\title{
A Comparison Study of the Optical Measurement Sensitivity of the Phototransistor and Photodiode
}

\begin{tabular}{|cc|}
\hline \multicolumn{1}{|c|}{ Mehmet Ali ÖZÇELİK $* 1$} \\
\hline${ }^{1}$ Gaziantep University, Gaziantep Merkez Kampüsü, 27310 Gaziantep, TURKEY \\
\hline \\
\hline Başvuru/Received: $20 / 04 / 2017 \quad$ Kabul/Accepted: $18 / 12 / 2017 \quad$ Son Versiyon/Final Version: $29 / 01 / 2018$ \\
\hline
\end{tabular}

\begin{abstract}
Depending on the amount of light they receive, the light-sensitive circuit elements generate electrical signals such as current, voltage, and resistance. Thus, these sensors are widely used in light measurement systems, light control systems, and opto-couplers. In this study underhand, the electrical values produced by the application of increasing light on the photodiodes and phototransistors are analysed. According to values obtained, the 9.44 standard deviation shows that photodiode has a better performance than phototransistor in terms of light sensitivity.
\end{abstract}

\section{Key Words}

" Photodiode, Phototransistor, Light Intensity, Lux-meter, Photo-current " 


\section{Introduction}

Day-time light is indispensable for indoor areas such as living areas, factories, offices and etc. However, it is also a known fact that most of the times, the amount of energy consumed in these areas is more than needed [Ghassan et a.(2015) and Özçelik(2016)]. This being so, controlling the light intensity of the ambient, and thus saving energy becomes vital [Juan et al.(2016), Toufiq etmal.(2016), Gentile et al.(2016)]. To measure and control light, sensors such as phototransistors, photodiode, LDR (Light Dependent Resistor), optocouplers and photocells are utilized [Fathabadi (2016), Kamran et al.(2011), Coşkun et al.(2011), Lau et al.(2006), Özçelik (2016)]. The variation in these sensors' reactions to the light generally result in resistance, current, and voltage. The different electrical signals that are produced by the light sensors based on the light intensity defines the sensor sensitivity. Which is vital for light control and specifically for light measurement tools [Phama and Suhb(2017)]. Practically, the more intense the light, the less resistance, the upper current or voltage, however, the relation between the electrical elements and lux for light sensors is different. Some sensors have a linear tendency in their reactions to the light while others show a different trend. The values generated by the sensors in line with the light intensity are input data of control systems like microcontrollers. Through a mathematical figuring, the data obtained generate an illumination information at the system's output or they help with the adjusting the lighting level of an illumination system. Thus, selecting and adopting these sensors to measure and control systems is crucial.

Photodiode and phototransistor are in the group of photo-detectors, which are widely used in light measurement, control, and detection systems. In this study, photodiode and phototransistor are covered in a closed environment or indoor area. Characteristic curves are created from these current values obtained by varying the light values on these sensors and sensitivities are evaluated.

A photodiode is a semi-conductor element whose reverse direction current increases with the light intensity.

Photodiodes are connected reversely to the circuit, that is to say, the negative (-) tip is connected to anode and the positive $(+)$ to the cathode. The neutral zone in the P-N junction area creates a potential, namely, barrier voltage. If silicon is used in the photodiode structure, barrier voltage is $0.7 \mathrm{~V}$. A small leakage current flows through the photodiode when there is no light. Modern day silicon photodiodes are made by planar diffusion and optical-filters [Oliveira et al. (2016), Vishay Semiconductors (2016)]

The picture and form of photodiode can be seen in Figure 1.

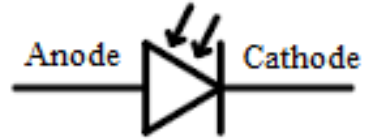

(a)

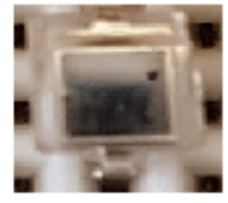

(b)

Fig.1. The form (a) and Picture (b) of the Photodiode

The area of use for photodiodes could be listed as in optocouplers, optical communications, lighting control, in the triggering of transistors, in counter circuits, in infrared remote control devices' receivers, sensor of lux-meter. Photodiodes or photoconductors could be used alternatively for many appliances. When the accurate measurement of light intensity is in question, photodiodes are often made, use of in science and industry and also for responding to the light levels, such as in turning on street light when it is evening.

Phototransistors are optoelectronic elements whose collector-emitter current is controlled by light. The bases of these transistors are not used, they are made of light-conducting or insulator. The working of the phototransistors is similar to NPN type and PNP type silicon transistors [Kostov et al. (2011)].

As a PIN of the phototransistors, which has high speed and is radiant-sensitive, a BP 103-5 has been used in this study, which is flat, top view, clear plastic package. It shows sensitivity to visible and close to infrared radiation [Osram(2017)].

Figure 2 shows the picture and form (symbol) of the phototransistor.

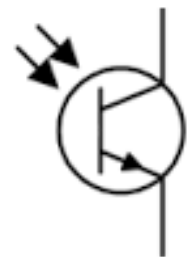

(a)

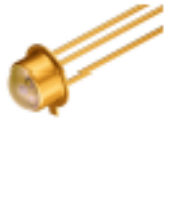

(b)

Fig.2. The form (a) and image (b) of the Phototransistor 
Phototransistors are light sensitive, photo-bipolar transistors are a popular kind of phototransistors which, by being placed in a transparent case enables light to reach the base-collector. It was first invented by Dr.John N. Shive in 1948. Phototransistors could be used in optical readers and level indicator devices as photodiodes as well. This is possible when the emitter is not connected while base and collector leads are utilized. Phototransistors could be distinguished from other transistors by the magnifier they have on them [Kostoy et al. (2011)].

The remaining part of the research is divided as follows: experimental part, in which general steps regarding the experiment have been tackled. Lastly, in the results and findings section, the values from photodiode and phototransistor depending on the light brightness (intensity) have been given. In the conclusion, remarks assessing the results obtained have been made.

\section{Experimental Setup}

The experimental unit set up to measure the optical sensitivity of the phototransistor and photodiode sensors will be investigated.

The experimental setup used in this research included a 5 V Direct Current (D.C.) electrical supply, Dimmer which is used to adjust the lighting level of a halogen lamp, $100 \Omega$ resistance, photodiode sensor, phototransistor sensor, multi-meter and for the indoor area lighting measurement DT-8809A professional lux meters (Max. range 400,000 lux, Resolution 0.1 lux, Accuracy $\pm \% 5$ ) have been used, The experimental mechanism is seen in Fig. 3.

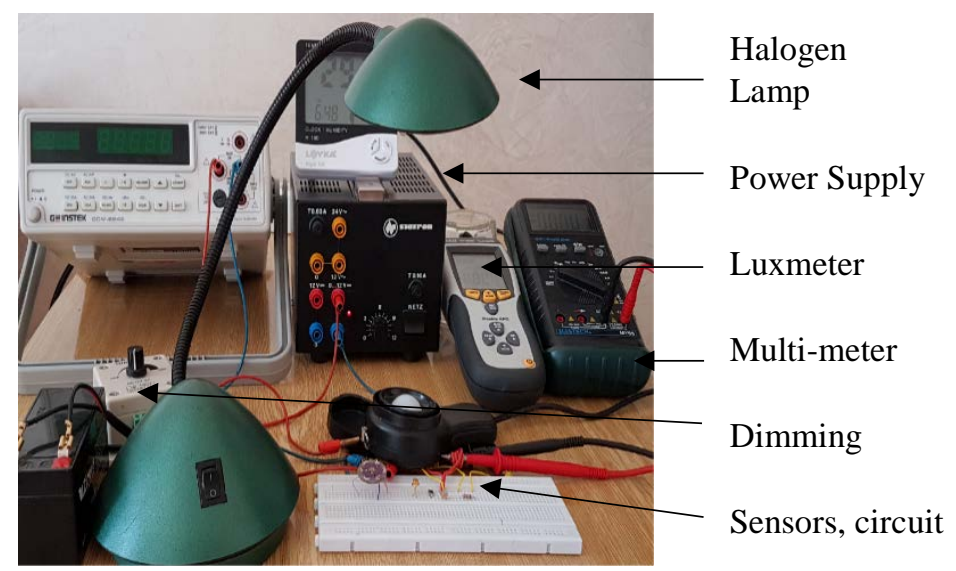

Fig.3. The experimental setup for measuring the electrical parameter of Photodiode and Phototransistor with changing light

\section{Result and Findings}

Two cases tackled to comparison both Photodiode and Phototransistor lighting sensors. Firstly,

\subsection{The Examine of Photodiode under Variable Illumination}

The photodiode is linked to $5 \mathrm{~V}, 100 \Omega$ resistor is applied to ground. In an indoor ambient, lighting level on photodiode is growing by 10 lux from 0 (dark) up to 150 (bright) lux. The current values at the output have been measured by digital multi-meter as it is shown in Figure 4.

Usually, in microcontrollers $+5 \mathrm{~V}$ is utilized, so this voltage rate has been considered

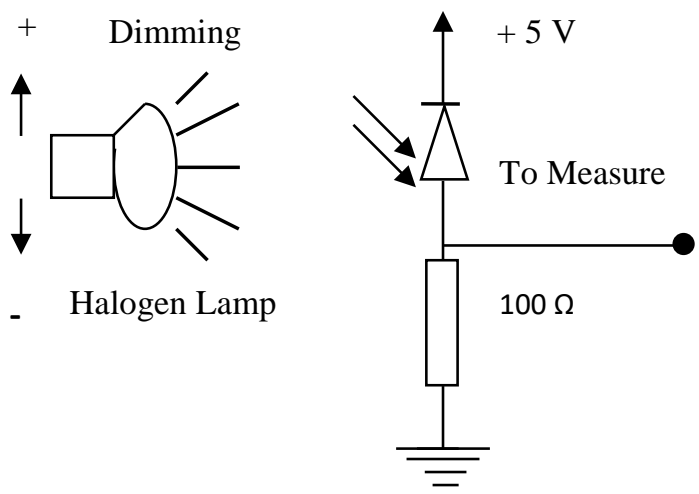

Fig.4. Variable Lighting Implemented on Photodiode

Figure 5 shows, photodiode's current changing curve concerning with the lighting rates growing from 0 to 150 lux. 


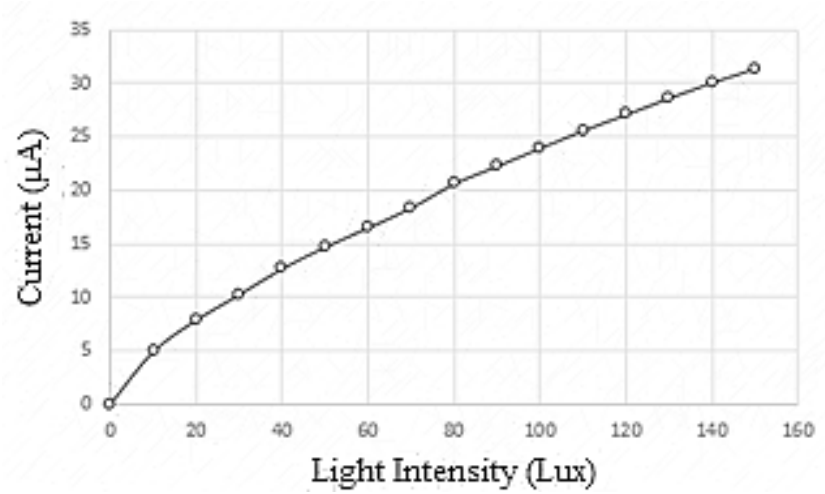

Fig. 5. Photodiode’s Current Output to the Illumination Varies

If Figure 5 is analysed, it is showed that while the light intensity rises, the current change rises exponentially. Depending on the illumination level, the current rates measure from ampere-meter have been seen in Table 1.

Table 1. Photodiode's Current Rate in Relation with Level of Lighting

\begin{tabular}{cc}
\hline Lighting Level (Lux) & $\begin{array}{c}\text { Current Rate of } \\
\text { Photodiode ( } \boldsymbol{\mu A})\end{array}$ \\
\hline 0 & 0 \\
10 & 4.92 \\
20 & 7.87 \\
30 & 10.27 \\
40 & 12.71 \\
50 & 14.72 \\
60 & 16.51 \\
70 & 18.37 \\
80 & 20.64 \\
90 & 22.28 \\
100 & 23.96 \\
110 & 25.62 \\
120 & 27.12 \\
130 & 28.66 \\
140 & 30.11 \\
150 & 31.41 \\
Maximum & 31.41 \\
Minimum & 0 \\
Mean & 18.44 \\
Standard Deviation & 9.44 \\
COV (Covariance (\%)) & 51.19 \\
SSE (the sum of the & 295.17 \\
squared errors) & 18.44 \\
MSE (the mean squared & 4.29 \\
error) & \\
RMSE (The root-mean- & \\
square deviation) & \\
\hline & \\
\hline
\end{tabular}

It is showed in Table 1 that the standard deviation value stands at 9.44. The root-mean square deviation (RMSE) rate is 4.29. 


\subsection{The Examine of Phototransistor under Variable Lighting}

In Figure 4, when phototransistor is connected in inverse bias in place of photodiode, Figure 6 comes about. Accordingly, when light intensity values from 0’ (dark) to 150 lux are applied to phototransistor, the curve in Figure 7 formed.

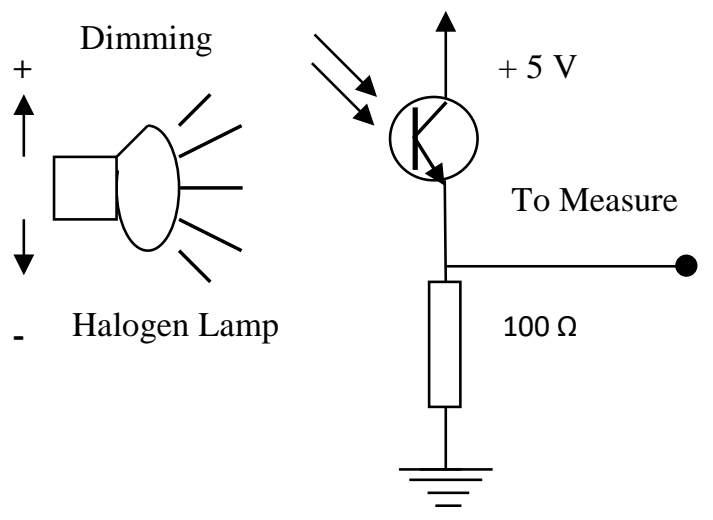

Fig.6. Variable Lighting Levels Being Implemented on Phototransistor

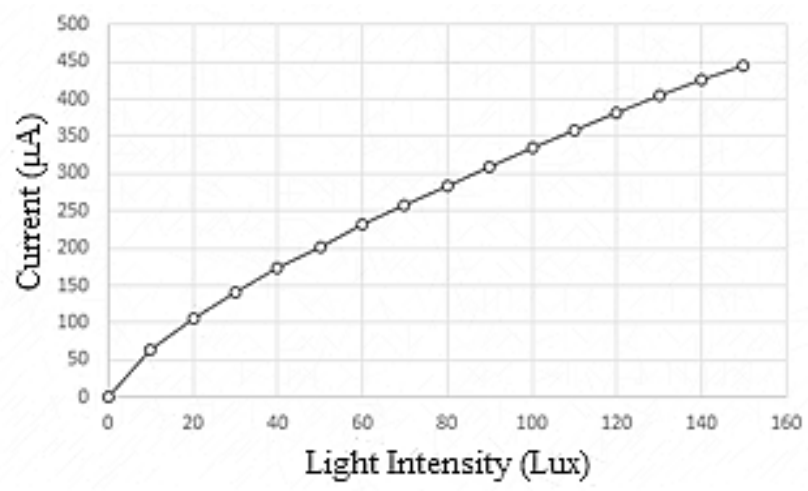

Fig. 7. Phototransistor's Current Rate to the Intensity of Light

If Figure 7 is analysed, it is realized that while the light level increases, so dues the current varied incrementally with bigger values than photodiode.

In association with the light level values applied to Figure 6, the measured current rates have been seen in Table 2.

Table 2. Phototransistor's Current Rate to Level of Lighting

\begin{tabular}{cc}
\hline Lighting Levels (Lux) & $\begin{array}{c}\text { Current Rate of } \\
\text { Phototransistor } \\
(\boldsymbol{\mu A} \mathbf{A})\end{array}$ \\
\hline 0 & 0.5 \\
10 & 63 \\
20 & 105.78 \\
30 & 141 \\
40 & 174.42 \\
50 & 202.13 \\
60 & 231.36 \\
70 & 258.14 \\
80 & 284.37 \\
90 & 309.36 \\
\hline
\end{tabular}




\begin{tabular}{cc}
\hline 100 & 333.45 \\
110 & 358 \\
120 & 380.35 \\
130 & 403.72 \\
140 & 425.33 \\
150 & 445.15 \\
Maximum & 445.15 \\
Minimum & 0.5 \\
Mean & 257.53 \\
Standard Deviation & 134.23 \\
COV (Covariance (\%)) & 52.176 \\
SSE (the sum of the & 4116.06 \\
squared errors) & \\
MSE (the mean squared & error) \\
RMSE (The root-mean- & 257.253 \\
square deviation) & 16.039 \\
\hline
\end{tabular}

As shown in Table 2, the Standard Deviation value stands at 134.23 and the root-mean square deviation (RMSE) value is 16.039.

Considering the results available from the two experiments, it can be realized that phototransistor's and photodiode's changes to light are linear. The current varies with 10 lux. But, in photodiode, the current variations are nearer lux than phototransistor.

\section{Conclusion}

In the study underhand, the current values from photodiode and phototransistors, which are known to be widely used in measurement and light control systems, have been tackled in on ambient with $28{ }^{\circ} \mathrm{C}$ temperature and $+5 \mathrm{~V}$ voltage under varying light brightness (intensity) and in line with this a sensitivity analysis has been conducted. Examining the variation obtained, it is noted that there is an exponential trend in both sensors' response to the light. However, the photodiode stand deviation is calculated to be 9.44 and phototransistor's is 134.23 . Combining all other data from the research, in terms of sensitivity and precision, photodiode draws a better profile.

\section{References}

Coskun, A., Sevil, H.E., Özdemir, S. (2011). Cost Effective Localization in Distributed Sensory Networks, Engineering Applications of Artificial Intelligence, 24, 232-237.

Gentile, N., Laike, T., Dubois, M.C. (2016). Lighting Control Systems in Individual Officies Rooms at High Latitude : Measurements of Electricity Savings and Occupants’ Satisfaction, Solar Energy, 127, 113-123.

Ghassan, M.S., Hashimah, I., Debnath, N., Nadya, A. (2015). Optimal Light Power Consumption Using LDR Sensor. 2015 IEEE International Symposium on Robotics and Intelligent Sensors (IEEE IRIS2015), 144-148.

Fathabadi, H. (2016). Comparative Study between Two Novel Sensorless and Sensor Based Dual-Axis Solar Trackers, Solar Energy, 138, 67-76, 2016.

Juan, F.D.P, Bajo J., Rodriguez, S., Villarubia G., Corchado J.M. (2016). Intelligent system for lighting control in smart cities. Information Sciences, 372, 241-255.

Kamran, A., Khokhar, A.Z., Rahman, F. (2011). High responsivity silicon MOS phototransistors, Sensors and Actuators A, 172, 434-439.

Kostov P, Hornstein K.S., Zimmermann H. (2011). Phototransistors for CMOS Optoelectronic Integrated Circuits, Sensors and Actuators, 172, 140-147.

Lau, K.T., Baldwin, S., O’Toole, M. (2006). A Low-Cost Optical Sensing Device Based on Paired Emitter-Detector Light Emitting Diodes, Analytica Chimica Acta 557, 111-116.

Oliveira, C.N.P., Khoury, H.J., Santos, E.J.P. (2016). PiN photodiode performance comparison for dosimetry in radiology applications Physica Medica, 32(12), 1495-1501. 
Osram. (2017). Phototransistor BP 103-5.

Özçelik, M.A. (2016). Light Sensor Control for Energy Saving in DC Grid Smart LED Lighting System Based on PV System. Journal of Optoelectronics and Advanced Materials, 18(5-6), 468-474.

Özçelik, M.A. (2016). The Analysis of the Optical Measurement Sensitivity of the Photodiode and LDR Light Sensors. International Energy\& Engineering Conference (UEMK 2016), 13-14 October, Gaziantep/Turkey, 688-695.

Phama, D.D., Suhb, Y.S. (2017). Remote length measurement system using a single point laser distance sensor and an inertial measurement unit, Computer Standards \& Interfaces, 50, 153-159.

Toufiq, I., Afshari, S., Sandipan, M. (2016). An Experimental Survey of Feedback Control Methodologies for Advanced Lighting Systems, Energy and Building, 130, 600-612.

Vishay Semiconductors (2016). BPW34, Document Number 81521. 